\title{
Une approche micro- mécanique de la notion de contrainte effective en mécanique des milieux poreux
}

\section{DORMIEUX}

Université Paris-Est

Institut Navier

Cité Descartes

Champs-sur.Marme

77454 Marne-la-Vallée

Cedex 2

domieux@msgc.enpc.fr

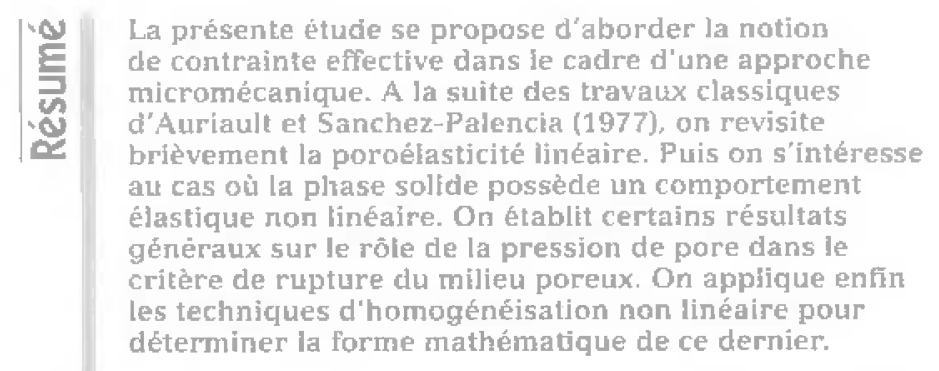

Mots-clés : contrainte effective, homogénéisation, non linéaire, rupture.
The present paper considers the effective stress concept in the framework of a micromechanics reasoning. Following the classical works of Auriault and Sanchez-Palencia I1977, tinear poroelasticity is first brietly revisited. Ther, the case of a non linere elastic behavior of the salid phase is examined. Some gereral results concerning the influence of the pore pressure or the strengh of a porous medium are presented. The strength. criterion is derived in particular cases by mesns of non Jiraat homogenization techniques.

Key words : effective stress, homogenization, non linear. strength. 


\section{Introduction}

I'introduction du concept de conliainte effective en mécanique des milieux poreux est due à $\mathrm{K}$. Terzagh et M. Biot qui l'ont formulé de façon indépendante dès la première moitie du $X X^{e}$ slècle. Il ne s'agit pas ici de faire l'historique de ce concept. Cependant, il est incontestable que son impact sur l'essor de la géotechnique a été décisif. Sur le pan théorique, il semble que la justitication de l'idée de contrainte effective ail d'abord été apportée dans le cadre de la théorie lineare de la poroélasticité, qui suppose que le comportement do la phase solide est élastique lnéaire. Cepenclant, de nombreux auteurs, notaminent à lis suite de Terzaghi, ont cherchể très tôt à l'utiliser dans le domaine du comportement irréversible de la phase solide et de l'analyse de la résistance des milieux poreux. Par ailieurs, alors qu'il avait été inftialement introduit pour des matériaux saturés par une phase fluide unique. le recours à ce concept dans des situations multiphasiques a également été envisagé sous diverses formes (Bishop, 1960; Fredlund, 1985 ; Coussy, 1995 ; Schrefler et al., 1997 ; Château et Dormieux, 2002).

La présente étude se propose d'aborder la notion de contrainte effective dans le cadre d'une approche micromécanique. A la suite des travaux classiques d'Auriaut et Sanchez-Palencia (1977), on revisite brièvement la poroélasticité linéaire. Puis, on s intéresse au cas où la phase solide possède un comportement élastique non linéaire. On établit centains résultats générabx sur le rôle de lá pression de pore dans la critère de rupture du milieu poreux. On appligue enfin les techniques d'homogénéisation non linéaire pour déterminer la forme mathematique de ce demier.

Le point de vue micromécanique consiste à considérer la particule élémentaje introduite classiquement par la mécanique des milieux continus comme une structure et à déduire le comportement du matériau de la réponse de cette structure torsqu'elle est soumise è un chargement défin de façon adéquate. Plus précisëment, la mise en ceuve du raisonnement dhomogénéisation dans les milieux hétérogènes à microstructure aléatoire repose sur la notion de volume élémentaire représentatif [v.e.r.]. Ce dernier incorpore de façon statistique l'ensemble des informations relatives à la géométrie de la microstructure et aux propriétés mécaniques des constituants. A cet effet, sa taille coit être grande devant celles des hétérogénéités (dimensions caractéristiques des pores et des grains).

Le ve.r. sur lequel on travaille dans la suite est un volume $\Omega$. I] est subdivisê en un sous-domaine solide et un espace poreux. Le vecteur position cans $\Omega$ à l'échelle microscopique est noté z. Les efforts intérieurs dans le ve.r. et les déformations qu'il subit sont décrits

- à l'échelle microscopique, par un champ de contrante $\sigma$ (z) et un champ de déformation $\varepsilon(z)$;

- à l"échelle macroscopique, par un tenseur de contrainte $\Sigma$ et un tenseur de déformation $\mathrm{E}$.

La configuration de reference du ver. correspond à l'ëtat raturel en l'absence de contrainte macroscom pique et de pression de Iluide dans l'espace poreux. $\Omega_{a}^{5}$ et $\Omega_{0}^{n}$ représentent les configulations de référence de la phase solide et de l'espace poreux. $\Omega_{n}=\Omega_{0}^{5} \cup \Omega_{\mathrm{f}}^{5}$ représente ainsi la configunation de référence du ve.r. et $\left|\Omega_{\mathrm{g}}\right|$ désigne le volume correspondant.
Sous l"action d'un chargement mécanique, le sousdomaine solide et l"espace poreux subissent une trans. fomation géométrique au terme de laquelle ils occupent respectivement les volumes $\Omega^{\circ}$ et $\Omega^{\text {e }}$. Ir désigne la position actuelie de l'nterface solide/pores. Dans toute la suite, on fait l'hypothese de transformations infïritésinales.

Sans restreindre la généralité, il est commode de supposer que la frontière extérleure ơu v.e.r. appartient à la phase solide. Pour rendre compte de la connexite de l"espace poreux, il sutitit de supposer que la pression de pore qui règne dans $\Omega^{p}$ est uniforme.

Pour la suite. il est commode d'introduire le rapport $\phi=\left|\Omega^{p} / / \Omega_{a}\right|$ gui foumit une valeur nomalisée du volume actuel de l'espace poreux. Dans la configuration de référence, $\phi=\phi_{\text {w }}$ n'est autre que la poroslté initiale $\Phi_{\circ}$. Cependant, les variations de $\phi$ sous l'action d"un chargement mécanique ne sont pas égales à celles de la porosité.

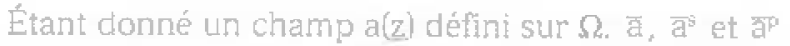
désignent les valeurs moyennes al sens intégral usuel du charnp a respectivenent sur $\Omega, \Omega^{5}$ et $\Omega$. On note 1 et I les tenseurs idertités đu second et du cuatrième ordre. On introduit également $\mathbb{i}=1 / 31 \otimes 1$ et $\mathrm{K}=\mathbb{I}-\mathrm{J}$.

\section{2}

\section{Poroélasticité linéaire}

Dans cette section, le comportement du solide est élastique linéaire. Les propriétés du solide, supposées homogènes, sont caractérisées par lé tenseur des modules d'élasticité $\mathbb{C}^{5}$, on par le tenseur de souplesse $S^{s}=\mathbb{C}^{s-1}$. Pour certaines illustrations, on se placera dans le cadre de l'isotropie, dans lequel $\mathbb{C}^{\mathrm{s}} \mathrm{s}^{\text {écrit }}$ :

$$
\mathrm{C}^{5}=3 \mathrm{k}^{\mathrm{g} J}+2 \mu^{\mathrm{s}}
$$

ob $k^{5}$ est le module de compression; $\mu^{*}$ le module de cisaillement.

\section{1}

\section{Définition du chargement}

Il existe classiquement deux approches posstbles pour définir le chargement mécanique subi par la phase solicte $\Omega^{5}$ du ve.r. (Zaoui, 2002). Lorsque l"on adopte les conditions aux limites dites un unformes en contraintes a le chargement macroscopique est caractérisé par le tensaur de contraintes macroscopique $\Sigma$ et la pression macroscopique P:

$$
\begin{array}{ll}
\operatorname{div}_{z} \sigma=0 & \left(\Omega^{s}\right) \\
\sigma=\mathbb{C}^{3}: \varepsilon & \left(\Omega^{y}\right) \\
\sigma \cdot \underline{\underline{H}}=\Sigma \cdot \underline{\underline{z}} & (\partial \Omega) \\
\sigma \cdot \underline{n}=-P_{\underline{I}} & \left(I^{y}\right)
\end{array}
$$

La seconde possibilité consiste à utiliser des conditiots dux limites uniformes en déformation, pour lesquelles les paramètres de chargement sont à présent le tenseur de céformation macroscopique $\mathbf{E}$ et la pression macroscopique ? 


$$
\begin{aligned}
& \operatorname{div}_{\underline{z}} \sigma=0 \\
& \sigma=\mathbb{C}^{s}: \varepsilon \\
& \underline{\xi}=\mathrm{E} \cdot \underline{z} \\
& \sigma \cdot \underline{n}=-P_{\underline{n}}
\end{aligned}
$$

Dans les équations (2) et (3), le système matêriel coinncide avec la phase solide. Cependant, il est commode d'étencre la définition du problème micromécanique à la totalité du volume élémentaire [solide et espace poreux saturé). Il suffit d'introduire un matériau fictif dans l'espace poreux, dont le comportement est de type élastique linéaire avec prêcontrainte:

$$
\sigma=\mathbb{C}^{\mu}: \varepsilon-p 1 \text { avec }\left|\mathbb{C}^{\prime}\right| \ll\left|\mathbb{C}^{*}\right|
$$

La condition $\mid \mathrm{C}$ p $<\left|\mathrm{C}^{s}\right|$ revient en fait à $\sigma \approx-\mathrm{P} 1$ dans $\Omega^{p}$ et rend compte de la condition de continuité à l'interface fluide-solide isf dans les équations (2) et (3). L'intérêt de ce point de vue réside dans le fait qu'il permet de formuler le comporternent local d"une façon unifiée pour" la totalité du ve.r.

$$
(\forall \underline{z} \in \Omega) \quad \sigma(\underline{z})=\mathbb{C}(\underline{z}): E(\underline{z})+\sigma_{0}(\underline{z})
$$

avec:

$$
\mathbb{C}(\underline{z})=\left\{\begin{array}{ll}
\mathbb{C}^{p} & \left(\Omega^{p}\right) \\
\mathbb{C}^{\star} & \left(\Omega^{*}\right)
\end{array} \quad \sigma_{\theta}(\underline{z})=\left\{\begin{array}{l}
-P 1 \\
0
\end{array}\right.\right.
$$

Ainsi, dans le cadre des conditions aux limites en contraintes, le problème trécanique (2) peut être étendu à la totalitié du v.e.r. $\Omega$ sous la forme:

$$
\begin{aligned}
& \mathrm{diF}_{a} \sigma=0 \\
& \sigma=\mathbb{C}(\underline{z}): \varepsilon+\sigma_{0}(\tilde{z}) \\
& \sigma \cdot \underline{\underline{z}}=\Sigma \cdot \underline{\underline{z}}
\end{aligned}
$$

Avec les conditions aux limites uniformes en déformation, l'équation (3) est maintenant remplacée par

$$
\begin{aligned}
& \mathrm{div}_{,} \sigma=0 \\
& \sigma=\mathbb{C}(\underline{z}): \varepsilon+\sigma_{o}(z) \\
& \overrightarrow{\underline{F}}=\mathbb{E} \cdot \underline{z}
\end{aligned}
$$

\section{9}

\section{Conditions drainées}

Pour commencer, on traite brièvement les cas de chargement définis respectivement par $(\Sigma \neq 0, P=0)$ et par $\left(\mathrm{E} \neq 0, \mathrm{P}=0\right.$ ). Cela revient à poser $\sigma_{01}=0$ dans (7) et (8) :

$$
\sigma=\mathbb{C}(\underline{z}): \varepsilon \quad \Leftrightarrow \quad \varepsilon=\mathbb{S}(\underline{z}): \sigma \text { avec } \mathbb{S}(\underline{z})=\mathbb{C}(\underline{z})^{-1}(9)
$$

Ausși bien dans l'équation (7) que dans l'équation (8), la réponse dépend linéairement du paramètre de chargement, c'est-à-dire de $\Sigma$ ou de $\mathbf{E}$. Cette remarque conduit au concept important de tenseur de ut localisation $\cdots$.

Plus précisément, considérons d'abord les conditions uniformes en contrainte (problème (7)). Le champ de contrainte $\sigma(z)$ dans le ve.r. est proportionnel au paramètre de chargement $\Sigma$. Il existe donc un tenseur du quatrième ordre, dit a tenseur de localisation de la contrainte s, noté ici $\mathbb{B}$ (z) (Fig. 1) :

$$
(\forall \underline{\underline{z}} \in \Omega) \quad \sigma(\underline{z})=\mathbb{B}(\underline{\underline{z}}): \Sigma
$$

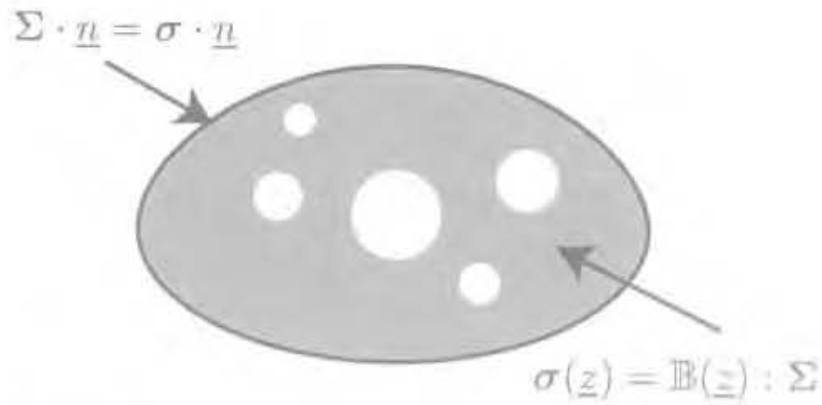

ก๔. Le tenseur $\bar{B}$ de localisation de la contrainte.

On note que la règle de moyenne sur les contraintes $\Sigma=\tilde{\sigma}$ qui résulte des conditions aux limites choisies implique que $\overline{\mathbb{B}}=\vec{I}$. En vertu de la condition $\mid \mathrm{C}$. $\& \mid \mathrm{CS}$, l'état de contrainte dans l'espace poreux est $\sigma=0$. Ceci revient à ecrire que | I Is $=0$ dans $\Omega^{p}$ et implique que :

$$
\mathbb{I}=\overline{\mathbb{B}}=\left(1-\varphi_{0}\right) \overline{\mathbb{E}^{s}}+\varphi_{u} \overline{\mathbb{B}}^{p} \approx\left(1-\varphi_{n}\right) \overline{\mathbb{E}}^{s}
$$

La déformation macroscopique $\mathrm{E}$ étant définie comme la moyenne $\bar{\varepsilon}$, la combinaison des équations (9) et (10) foumit :

$$
\mathrm{E}=\mathbb{S}^{\text {hom }}: \Sigma
$$

avec:

$$
\mathbb{S}^{\text {hom }}=\overline{\mathbb{S}: \mathbb{B}}
$$

L'équation (12) montre que le comportement macroscopique est linéaire élastique et shom s'interprète comme le tenseur de souplesse homogénéisé. Les tenseur's de souplesse $\mathbb{S}^{5}$ du solide et $\mathbb{S}^{p}$ de l'espace poreux (rappelons le caractère fictif de ce dernier) étant uniformes, Șiont şécrit:

$$
\mathbb{S}^{h a m}=\left(1-\varphi_{0}\right) \mathbb{S}^{s}: \overline{\mathbb{B}^{9}}+\varphi_{0} \mathbb{S}^{p}: \overline{\mathbb{B}}^{2}
$$

soit, en se souvenant de la relation (11):

$$
\mathbb{S}^{\text {hom }}=\mathbb{S}^{s}+\varphi_{0} \mathbb{S}^{p}: \overline{\mathbb{R}^{p}}
$$

La souplesse macroscopique apparaît comme la somme des contributions respectives du solide et de l'espace poreux. Bien que $\bar{B}^{P} \approx 0$, le second terme de

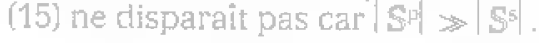

De façon similaire, dans le cadre des conditions uniformes en déformation (8), le champ de déformation microscopique $\varepsilon$ dépend linéairement de $\mathbf{E}$. Cette propriêté est prise en compte par l'intemédiaire du tenseur de localisation de la déformation $A(\underline{\underline{z}})$ (Fig. 2) :

$$
(\forall \underline{z} \in \Omega) \quad \varepsilon(\underline{z})=\mathbb{A}(\underline{z}): \mathbb{E}
$$

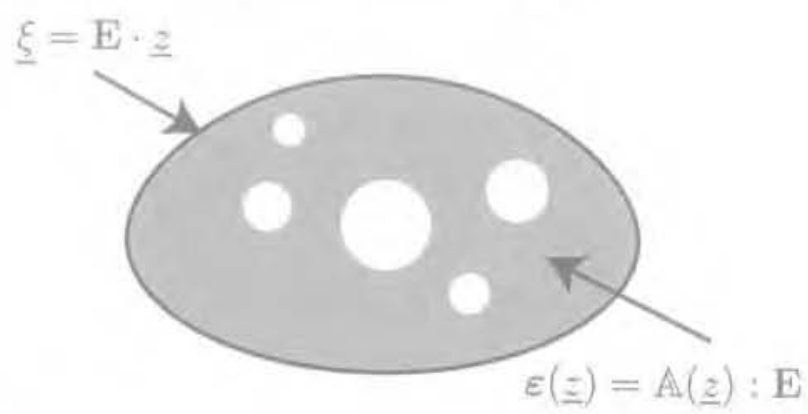

Mis. . Le tenseur $\mathbb{A}$ de localisation de la dêformation .

La règle de moyenne $\mathbf{E}=\bar{\varepsilon}$ qui résulte de ce type de conditions aux limites implique cque $\overline{\mathbb{A}}=\mathbb{I}$ :

$$
\mathbb{I}=\left(1-\varphi_{0}\right) \overline{\mathbb{A}}^{y}+\varphi_{n} \overline{\mathbb{A}}^{p}
$$


On définit alors la contrainte macroscopique comme Ia movenne du champ de contrainte microscopique: $\Sigma=\bar{\sigma}$. En vertu des équations (9) et (16), il résulte que :

$$
\Sigma=\mathbb{W}^{\text {hom }}: \text { E } \text { avec } \mathbb{C}^{\text {hom }}=\overline{\mathbb{C}: A}
$$

Cham s'interprète comme le tenseur des modules d'élasticité en condition drainée. En utilisant l'équation (17) et en se souvenant du fait que $\mid \mathbb{C}$. $\rightarrow 0$, il peut être mis sous la forme :

$$
\mathbb{C}^{\text {ham }}=\left(1-\varphi_{n}\right) \mathbb{C}^{b}: \overline{\mathbb{A}}^{8}=\mathbb{C}^{s}:\left(\mathbb{I}-\varphi_{0} \overline{\mathbb{A}^{p}}\right)
$$

Le terme $\varphi, \overline{A^{p}}$ dans lexpression (19) de Chom rend compte de la reduction de raicleur imputable poreux.

D'une part, les conditions aux limites uniformes en contrainte conduisent au tenseur de souplesse homogénéisé Snom: D'autre part, le tenseur des modules délasticité macroscopiçue Com a été obtenu dans le cadre des conditions aux limites uniformes en déformation. La cohérence de ces deux différentes technjques réside dans le fait que les conditions aux limites correspondantes induisen! des réponses identiques à l'échelle locale, à l'exception du voistnage de la frontière $\partial \Omega$, sur une épaisseur de l'ordre de la taille des hétérogéréités. Cela revient à dire que Sthon = Cham-11 pourvu que la taille du ve.r. soit grande devant celles des hétérogénéités. Plus précisément, Hill et Mandel ont prouvé que :

$$
\mathbb{S}^{\text {ham }}: \mathbb{C}^{\text {hum }}=\mathbb{I}+\mathcal{O}\left(\frac{d}{\ell}\right)^{3}
$$

ò d et $\ell$ désignent respectivement la tailla des hetérogếrétiés et celle du ver.

\section{3}

\section{Le couplage poroélastique linéaire}

Le problème (7), défini par $\Sigma$ et $P$, dépend linéairement de ces deux paramètres de chargement. II est donc possible d'étudier la réponse au chargement $L_{1}=$ $(\Sigma, P)$ en le scindant en deux composantes, respectivement $\Sigma_{1}=[\Sigma+\mathrm{P} 1,0]$ et $L_{-}=(-\mathrm{P} 1, \mathrm{P})$.

Dans le chargement $L_{\nu}$, la contrainte macroscopique appliquée à la frontière $2 \Omega$ est $\Sigma+$ P1 et il n'v a pas de pression dans l'espace poreux. La réponse à un tel chargement a été déterminée au paragraphe 2.2. Les champs de contrainte et de déformation microscopi= ques s'écrivent

$$
\begin{gathered}
(\forall \underline{z} \in \Omega) \quad \sigma_{1}=\mathbb{E}:(\Sigma+P 1) \\
E_{1}=S(z): \mathbb{E}:(\Sigma+P 1)
\end{gathered}
$$

Dans le chargement $\mathcal{L}_{v}$ une même pression P est appliquée sur la frontiere da et sur l'jnterface fluidesolide 's'. Ainsi, le domatne solide $\Omega^{7}$ est soumis à une pression uniforme sur l'intégralité de sa frontière. Puisque le solide est supposé homogène, les champs microscopiques de contrainte et de déformation dans $\Omega^{5}$ sont uniformes et peuvent être étendus avec les mèmes valeurs à lespace poreux :

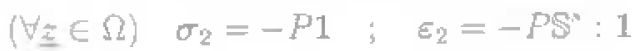

En vertu de la linéarité, la réponse au chargement $L=L_{1}+L_{2}$ est :

$$
\begin{gathered}
(\underline{\underline{z}} \in \Omega) \quad \sigma=\mathbb{B}:(\Sigma+P I)-P 1 \\
E=\mathbb{S}: \mathbb{B}:(\Sigma+P 1)-P S: 1
\end{gathered}
$$

Compte tenu de la relation (13), la déformation macroscopicue $E=\bar{E}$ se met sous la forme:

$$
\mathrm{E}=\mathrm{S}^{\text {hom }}:(\mathbf{\Sigma}+\mathrm{P} \mathbf{1})-P \mathrm{~S}^{\mathrm{s}}: \mathbf{1}
$$

ou encore

$$
\mathrm{E}=\mathrm{S}^{\text {hon }}:(\Sigma+P \mathrm{~B}) \Leftrightarrow \Sigma=\mathbb{C}^{\text {hath }}: \mathrm{E}-\mathrm{BP}
$$

avec :

$$
\mathrm{B}=1-\mathbb{C}^{\text {homm }}: \mathrm{S}^{5}: 1
$$

La relation (25) montre que le tenseur $\Sigma+P B$ contrôle la déformation macroscopique jnduite par $L$ et porte à ce tilíse le nom de a contrainte effective $y$. Cette relation constitue la première équation d'état du comportement poroélastique. La forme isotrope de [26] mérite d’être notée. Elle fait intervenir le module de compression homogénélsé knom et le module de compression $k^{5}$ du snlide (voir (1)\}:

$$
\mathrm{B}=b 1 \text { avec } b=1-\frac{k^{\text {ham }}}{k^{5}}
$$

On note que la contrainte effective de Biot $\Sigma+$ PB tend vers celle de Terzaghi, c'est-à-dire $\Sigma+P 1$ dans la limite $\mathrm{k}^{\mathrm{hom} / \mathrm{K}^{\mathrm{k}} \rightarrow 0} \rightarrow$

La seconde équation d'état concerne les variations du volume des pores, décrites par celles du volume normalisé $\$$. Le point de départ consiste à observer ģue:

$$
\phi-\phi_{0}=\phi_{u} 1 ; \vec{\varepsilon}^{2}
$$

On tire siors de la relation (23) que

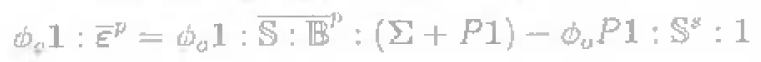

$\phi_{\varphi} 1: \overline{\mathrm{S}}^{3}$ peut être éliminé à l'aide de la relation (15)

En introduisant (25) et (26) dans (29), on obtient finalement:

$$
\phi-\phi_{o}=\mathrm{B}: \mathrm{E}+\frac{P}{N}
$$

ดั่ :

$$
\frac{1}{N}=\left(\mathrm{B}-\phi_{0} 1\right): \mathrm{S}^{*} ; 1
$$

A l'aide de la formule (27), on montre la forme isotrope de l'équation (31):

$$
\frac{1}{N}=\frac{b-\phi_{0}}{k_{i}^{*}}
$$

les équations [25] et (30) constituent la base de la théorie poroélastique linéaire de Biot. Il est rema.tudable que les coefficients poroélastiques $\mathbf{B}$ et $\mathbf{N}$ de (26) et (31) puissent être déteminés à partir des propriétés élastiques du solide et de celles du matếriau poreux drainé, regroupées dans le tenseur $\mathbb{G}^{\text {hom }}$

\section{4}

\section{Approche énergétique}

On raisonne ci-après dans le cadre des conditions aux limites uniformes en déformation. La puissance mécanique W fournie à la phase solide $\Omega^{5}$ sous l'action du taux de chargement ( $\dot{E}, \overline{\text { pl }}$ comporte deux contributions:

- la puissance de la pression de pore agissant sur l'interface $1^{\text {st }}=\partial \Omega^{\Gamma} \cap \partial \Omega^{*}$

- la puissance des forces surfaciçues o a agissant sur la frontiere $\partial \Omega n d \Omega^{5}$ dans la vitesse E.z qui est prescrite à cette interface : 


$$
\dot{W}=\int_{\partial \Omega} \underline{z} \cdot \dot{\mathrm{E}} \cdot(\sigma \cdot \underline{n}) d S+\int_{\left.F^{*}\right\}}-P_{\underline{n}} \cdot \underline{\xi} d S
$$

où n désigne le vecteur unitaire normal à $\partial \Omega^{\mathrm{s}}=\partial \Omega \cup \mathrm{IIr}^{\mathrm{s}}$, orienté vers l'extérieur du solide $\Omega$ s.

En invoquant l'équation de l'équilibre div $\sigma=0$, la première intégrale du membre de droite devient:

$$
\dot{E}_{i j} \int_{\partial n} z_{j} \sigma_{i k} n_{k} d S=\dot{E}_{i j} \int_{\Omega} \sigma_{i j} d V_{z}=\overline{\sigma_{i j}} \dot{E}_{i j}\left|\Omega_{0}\right|
$$

Par ailleurs, le fiux de la vitesse fluide-solide dans la deuxième intégrale n'est autre que le taux de vartation du volume des pores:

$$
\int_{i+1}^{\infty}-\underline{n} \cdot \underline{\dot{\xi}} d S=\left|\Omega_{0}\right| \dot{\phi}
$$

Combinant les relations (34) et (35) dens (33), la puissance fournie au domaine solide $\Omega^{5}$ est :

$$
\dot{W}=\left|\Omega_{0}\right|\left(\Sigma_{i,} E_{i j}+P \phi\right)=\left|\Omega_{0}\right|(\Sigma: \dot{E}+P \phi)
$$

On introduit les densités volumiques d'énergie élastique $\Psi$ (E, P) et d'ênergie potentielle $\Psi^{*}\left(E_{\text {, }}\right.$ PT relatives à la phase solide du ve.r. Elıes sont toutes deux fonctions de $\mathbf{E}$ et $P$ :

$$
\begin{gathered}
\Psi(\mathrm{E}, P)=\frac{1}{2\left|\Omega_{\theta}\right|} \int_{\Omega^{*}} \varepsilon: \mathbb{C}^{s}: \varepsilon d V \\
\Psi^{*}=\Psi-P\left(\phi-\phi_{a}\right)
\end{gathered}
$$

En l'absence de mëcanismes dissipatifs, le travail fournf au solide est stocké sous forme d'énergle élastique : $W=\left|\Omega_{0}\right| \Psi$. Il en résulte que :

$$
\dot{\Psi}^{*}=\Sigma: \dot{\mathrm{E}}-\left(\phi-\phi_{0}\right) \dot{P} \Rightarrow\left\{\begin{array}{l}
\boldsymbol{\Sigma}=\frac{\partial \Psi^{*}}{\partial \mathrm{E}} \\
\phi-\phi_{\theta}=-\frac{\partial \Psi^{*}}{\partial P}
\end{array}\right.
$$

Les relations (38) font apparaitre que l'énergie potentielle $\Psi^{*}$ est un potentiel thermodynamique pour le choix des variables d'état $E$ et $P$. En vertu de la linéaritè des équations d'état macroscopiques (25) et (30), "\$* est une fonction quadratigue de ses arguments $\mathbf{E}$ et $P$ :

$$
\Psi^{*}=\frac{1}{2} \mathrm{E}: \mathbb{C}^{\text {hiom }}: \mathrm{E}-\frac{P^{2}}{2 N}-P \mathrm{~B}: \mathrm{E}
$$

La combinaison de (37) et de (39) conduit à l'expression suivante de $\Psi$ :

$$
\Psi=\frac{1}{2} \mathrm{E}: \mathbb{C}^{\text {huer }}: \mathrm{E}+\frac{P^{2}}{2 N}
$$

\section{5}

\section{Niveau de déformation moyen dans la phase solide}

En vue d"applications ultérieures, il est intéressant d'évaluer le niveau de déformation local dans la phase solide en fonction du chargement macroscopique, définí par $\mathbf{E}$ et $\mathrm{P}$, ou par $\Sigma$ et $\mathrm{P}$.

Considérons pour commencer la déformation moyenne $\bar{\varepsilon}^{s}$, obtenue à partir de la moyenne de l'équation d'état locale $\varepsilon=\mathcal{S}^{s} ; \sigma$ dans le solide :

$$
\bar{\varepsilon}^{*}=\S^{5}: \bar{\sigma}^{*}
$$

La règle de moyenne des contraintes donne par ailleurs :

$$
\Sigma=\left(1-\phi_{\mathrm{o}}\right) \bar{\sigma}^{n}-\phi_{0} P 1
$$

En combinant [41] avec (42), on obtient une expression de la déformation moyenne en fonction de ba contrainte macroscopique et de la pression de pore:

$$
\left(1-\phi_{0}\right) \bar{\epsilon}^{s}=\mathbb{S}^{s}:\left(\Sigma+P \phi_{a} 1\right)
$$

If convient d'observer que $\bar{\varepsilon}^{s}$ est contrôlée par la contrainte effective $\Sigma+P \phi$ t. Si on décompose la déformation microscopique et sa moyenne dans la phase solide selon leurs composantes sphérigue et déviatorique, on obtient

$$
\varepsilon=\varepsilon_{d j}+\frac{1}{3} \varepsilon_{k} 1 \quad ; \quad \vec{E}^{\prime}=\overrightarrow{\varepsilon_{d i}}+\frac{1}{3} \overrightarrow{\varepsilon_{x}} 1
$$

avec $E_{\mathrm{y}}=$ tr $\mathrm{e}$. Dans le cas d'un comportement isotrope de la phase solide, la relation (43) donne:

$$
\begin{aligned}
& \left(1-\phi_{i}\right) \bar{\varepsilon}_{t}^{n}=\frac{1}{3 k^{t}} \operatorname{tr}\left(\Sigma+P \phi_{i} 1\right) \\
& \left(1-\phi_{i}\right) \bar{\varepsilon}_{d i}^{*}=\frac{1}{2 \mu^{\prime}} \Sigma_{d}
\end{aligned}
$$

oủ $\Sigma_{j}=\mathbb{R}: \Sigma$ est le déviateur de la contrainte macroscopique.

La formule (45) exprime que la moyenne de la déformation déviatorique $\bar{\varepsilon}_{\mathrm{d}}{ }^{5}$ n'est affectée que par $\Sigma_{\mathrm{d}}$ Ce résultat peut être illustré sur l'exemple d'une sphẻre creuse soumise à une contrainte de confinement un'forme sur sa frontière extérieure. La déformation déviatorique induite par ce chargement dans la phase solide est une composante essentielle du mécanisme de déformation de la sphère. Cependant, par raison de symétrie, la moyenne $\overline{\varepsilon_{d}^{3}}$ est nécessairement nulle. Cette remarque suggère qu'une telle quantte ne fourrit pas une estimation adéquate du niveau de déformation déviatorique dans la phase solide. Pour faire face à cette difficulté, on considère la grandeur scalaire $\varepsilon_{\mathrm{d}}$ ' dite déformation déviatorique équivalente

$$
\varepsilon_{d}=\sqrt{\frac{1}{2} \varepsilon_{d i}: \varepsilon_{d}}
$$

On se limite ci-après au cas où la phase solicle est isotrope (voir (1)]. Dans ce cas, la moyenne quadratique $E^{2 s}$ est reliée à la composante déviatorique de l'énergie élastique dans la phase solide. On dispose ainsi d'une mesure énergétique de la déformation déviatorique dans te solide. Celle-ci est exempte des limitations de $\overline{\varepsilon_{\mathrm{d}}^{\mathrm{s}}}$. On va voir qu'elle peut être évaluée à partir de la dérivée de l'énergie potentielle de la phase solide par rapport au module de cisaillement $\mu^{5}$ de cette dernière (Kreher, 1990). Rappelons que l'énergie potentielle de la phase solide s'écrit (voir (37)):

$$
\left|\Omega_{o}\right| \Psi^{*}=\frac{1}{2} \int_{\Omega^{*}} \varepsilon: \mathbb{C}^{\delta}: \varepsilon d V-P \int_{\Omega^{t}} \operatorname{tr} \varepsilon d V
$$

On note que $\Psi^{* *}$ dépend de $\mu^{s}$ explicitement à travers le tenseur $\mathbb{C}^{5}$ et implicitement à travers le champ de déformation microscopique $\varepsilon$. Observant que $\partial \mathbb{C}^{5} / \partial \mu^{s}=2 \mathbb{R}^{m}$ (cf (1)), il est facile de voir que

$$
\begin{gathered}
\left|\Omega_{n}\right| \frac{\partial \Psi^{*}}{\partial \mu^{*}}=\int_{\Omega^{*}} \varepsilon_{d}: \varepsilon_{d} d V+\int_{\Omega^{*}} \frac{\partial \varepsilon}{\partial \mu^{s}}: \mathbb{C}^{*}: \varepsilon d V+ \\
\int_{\Omega^{s}}-P 1: \frac{\partial \varepsilon}{\partial \mu^{*}} d V
\end{gathered}
$$


ou encore:

$$
\frac{\partial \Psi^{*}}{\partial \mu^{\circ}}=2\left(1-\phi_{0}\right){\overline{E_{d}^{2}}}^{*}+\overline{\sigma: \frac{\partial \varepsilon}{\partial \mu^{\sigma}}}
$$

Le lemme de Hill (voir annexe) peut ètre appliqué au couple $\sigma^{*}=\sigma$ et $\mathrm{E}^{*}=\partial \mathrm{d} / \mathrm{\mu}^{\mathrm{s}}$. En effet, le déplacement associé à $\partial \mathrm{e} / \partial \mathrm{J}^{\mathrm{s}}$ est $\xi^{*}=\partial \mathcal{E} / \partial \mu^{s}$ et ce dernier vérifie des conditions aux limites uniformes en deformation $\left[\mathrm{E}^{*}=0\right.$ ], de sorte que le second teme du membre de droite dis parait. Avec (39), on obtient donc:

$2\left(1-\varphi_{n}\right) \overline{\varepsilon_{i}^{*}}=\frac{\partial}{\partial x^{*}}\left(\frac{1}{2} \mathrm{E}: \mathbb{C}^{\text {hum }}+\mathrm{E}-\frac{p^{2}}{2 N}-P \mathbf{B}: \mathbf{E}\right)(50)$

Il est possible de simplifier encore cette expression dans le cas isotrope. Avec l"aide de (27) et (32), les dér" vées de $\mathrm{B}$ et ce $1 / \mathrm{N}$ s'écrivent

$\frac{\partial}{\partial \mu^{*}}(\mathrm{~B})=-\frac{1}{k^{*}} \frac{\partial k^{\text {hom }}}{\partial \mu^{2}}-1 ; \frac{\partial}{\partial \mu^{2}}\left(\frac{1}{N}\right)=-\frac{1}{k^{2}} \frac{\partial k^{2}}{\partial \mu^{2}}(51)$

En introduisant (51) dans (50), on obtient finalement: $2\left(1-Q_{a}\right) \overline{\varepsilon_{d}^{2}}=\frac{1}{2} \frac{\partial k^{\text {hiom }}}{\partial \mu^{*}}\left(\mathrm{tr} \mathrm{E}+\frac{P}{k^{*}}\right)^{2}+\frac{\partial \mu^{\text {ham }}}{\partial \mu^{*}} \mathrm{E}_{d}: \mathrm{E}_{d}$

De façon équivalente, l"équation d"état (25) peut être utilisée pour obtenir une expression de $\overline{\varepsilon_{d}^{2}}$ en fonction de la contrainte macroscopigue et de la pression de pore. En utilisant la relation de Biot (27) relative au coeficiciert $b$, on décompose (25) selon ses parties sphérique et déviatorique:

$$
\Sigma_{m}+P=k^{h m m}\left(\operatorname{tr} \mathrm{E}+\frac{P}{h^{-}}\right) \quad ; \quad \Sigma_{d}=2 \mu^{h o m} E_{d}
$$

où la contrainte moyenne $\Sigma$ et les contrainte et déformation déviatoriques équivalentes sont définies par:

$$
\begin{gathered}
\Sigma_{m}=\frac{1}{3} \operatorname{tr}(\Sigma) ; \quad \Sigma_{d}=\sqrt{\frac{1}{2} \Sigma_{d}: \Sigma_{d}} \\
E_{d}=\sqrt{\frac{1}{2} \mathrm{E}_{d}: \mathrm{E}_{d}}
\end{gathered}
$$

En reportant (53) dans (52), il wient:

$4\left(1-\phi_{0}\right) \overline{\Sigma_{1}^{2}}=-\frac{\partial}{\partial \mu^{2}}\left(\frac{1}{k^{\text {hom }}}\right)\left(\Sigma_{m}+P\right)^{2}-\frac{\partial}{\partial \mu^{2}}\left(\frac{1}{\mu^{i m m}}\right) \Sigma_{d}^{2}(55$

Tandis que la moyenne tensorielle de la déformation déviatorique $\overline{E_{d}}$ est indépendante de la pression de pore (voir (45)), il apparât' gue la moyenne guadratigue scalaire $\bar{c}^{25}$ dépend de la pression de pore à travers la contrainte effective de Terzaghi $\Sigma+P 1$.

La mểne démarche peut être appliquée à la détermination de la moyenne quadratique $\varepsilon_{\text {s. }}^{2 s}$. En particulier, on montre que l"équation homologue de (50) est:

$\frac{1}{2}\left(1-\phi_{u}\right) \bar{\varepsilon}_{v}^{*}=\frac{\partial}{\partial k^{*}}\left(\frac{1}{2} \mathrm{E}: \mathbb{C}^{\text {lowi }}: \mathrm{E}-\frac{P^{2}}{2 N}-P \mathrm{~B}: \mathrm{E}\right)(56)$

\section{3}

\section{Poroélasticité non linéaire}

\section{1}

\section{La méthode sécante}

On s'intéresse à présent à la situation où le comportement de la phase solide est élastique non linéaire. Il est caractérisé par un potentiel $\psi^{5}(\varepsilon)$ à l'alde duquel l'équation d'état du solide s'écrit:

$$
\sigma=\frac{\partial u^{s}}{\partial \varepsilon}
$$

$\Psi^{5}(\mathrm{c})$ représente physiquement la densité d'énergie libre du solide.

On suppose de plus que (57) peut se mettre sous la forme :

$$
\sigma=\mathbb{C}^{*}(\varepsilon): \varepsilon
$$

$\mathbb{C}^{\mathrm{s}}(\varepsilon)$ est appelé tenseur d'élasticité sécant.

Par exemple, si $\psi^{n}[\varepsilon]$ dépend de $\varepsilon$ à travers la déformation volumique $\varepsilon_{v}=$ tr $\varepsilon$ et l'invariant déviatorique $\varepsilon_{\text {. }}$ introduit en (46), $\psi^{*}=\psi^{s}\left(\mathrm{c}_{\mathrm{w}}, \mathrm{\varepsilon}_{\mathrm{c}}\right],(57)$ devient :

$$
\sigma=\frac{\partial v^{*}}{\partial \varepsilon_{z}} 1+\frac{1}{2 \varepsilon_{d}} \frac{\partial y_{i}^{*}}{\partial \varepsilon_{d}} \varepsilon_{d}
$$

On observe que (59) est bien de la forme (58) avec:

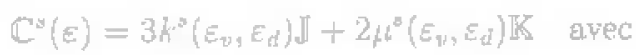

$$
\begin{aligned}
& \left\{\begin{array}{l}
k^{*}=\frac{1}{\varepsilon_{v}} \frac{\partial v^{\varepsilon}}{\partial \varepsilon_{v}} \\
\mu^{*}=\frac{1}{4 \varepsilon_{d}} \frac{\partial v^{*}}{\partial \varepsilon_{d}}
\end{array}\right.
\end{aligned}
$$

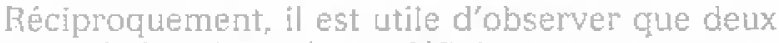
fonctions $k^{5}\left(\varepsilon_{\gamma}, \varepsilon_{c}\right)$ et $\mu^{5}\left(\varepsilon_{y}, \varepsilon_{d}\right)$ définissent un comportement élastique non linéaire a la condition que:

$$
\varepsilon_{t} \frac{\partial h^{*}}{\partial \varepsilon_{d l}}=4 \varepsilon_{d} \frac{\partial \mu^{*}}{\partial \varepsilon_{v}}
$$

On raisonne par exemple avec des conditions aux limites uniformes en déformation. Le chargement est donc défini par le tenseur de déformation macroscopique $\mathbf{E}$ et la pression de pore P. Les champs microscopiques de contrainte, de déformation et de déplacement $\sigma . \varepsilon$. E dans le v.e.r. sont solutions du problème suivant:

$$
\begin{aligned}
& \operatorname{div} \sigma=0 \\
& \sigma(\underline{z})=\mathbb{C}(\underline{z}): \varepsilon(z)+\sigma_{0}(\underline{z}) \\
& E=\frac{1}{2}(\operatorname{grad} \underline{\xi}+\operatorname{grad} \xi) \\
& \underline{\xi}(\underline{z})=\mathrm{E} \cdot \underline{z}
\end{aligned}
$$

où :

$$
\begin{gathered}
\begin{cases}\underline{z} \in \Omega^{*} ; & \mathbb{C}(\underline{z})=\mathbb{C}^{\prime \prime}(\varepsilon(\underline{z})) \\
\underline{z} \in \Omega^{*} ; & \mathbb{C}(\underline{z})=\mathbb{C}^{p}\end{cases} \\
\sigma_{0}(z)= \begin{cases}0 & \left(\Omega^{*}\right) \\
-P 1 & \left(\Omega^{p}\right)\end{cases}
\end{gathered}
$$

(62) est formellement identique fait que (6) est maintenant remplacé par (63). Rappelons que $\mathrm{G}^{\mathrm{l}} \rightarrow 0$

Il y a cependant une différence essentielle entre le problème linéaire (8) et le problème non linéaire (62): constant dans le premier cas, le tenseur d'élasticité local $\mathbb{C}^{5}\{\varepsilon(z)]$ dépend en revanche de la position dans la phase solide et du niveau de chargement dans le cas non linéaire. Maiheureusement, les schémas d'homogénéisation utilisés classiquement pour les milieux désordonnés ne sont pas capables de faire face à ce type d'hétẻrogénéité. Il est donc nécessaire de simplifier le problème. 
L'idée consiste à introduire un niveau de déformațion dit u effectif o destiné à prendre en compte la non linéarité de façon approchée (Suquet, 1997). Notée $\mathrm{e}^{\mathrm{e}}$, de nature tensorielle ou scalaire, il est naturel de s'attendre à ce que cette déformation effective soit une moyenne bien choisie du champ de déformation microscopique dans le solicie. Elle est donc a priori fonction du chargement macroscopicue $(E, P)$ ou $(\Sigma, P)$. La simplification réside dans le fait de remplacer l’élasticité hétérogène $\mathbb{C}(\varepsilon[\mathrm{z})]$ par une estimation uniforme :

$$
\left(\forall \underline{z} \in \Omega^{B}\right) \quad \mathbb{C}^{g}(\varepsilon(2)) \approx \mathbb{C}^{g}\left(\varepsilon^{e f}\right)
$$

Dans le cadre de (64), on est formellement ramené au cadre linéaire. On retrouve donc les équations d'état (25) et (30), à ceci près que les coefficients poroélastiques sont fonctions de $\varepsilon^{\text {t? }}$.

$$
\mathbb{C}^{\text {hom }}=\mathbb{C}^{\text {hown }}\left(\varepsilon^{\mathrm{J}}\right): \mathrm{B}=\mathrm{B}\left(\varepsilon^{\mathrm{e}}\right) ; N=\mathbb{N}\left(\varepsilon^{\mathrm{e} /}\right)
$$

Il convient alors de déterminer la dépendance de la déformation effective retenue en fonction des paramètres de chargement, par exemple $(\Sigma, P)$. $\varepsilon^{\text {ef }}$ se présente comme la solution d'un problème non linéaire de la forme suivante

$$
\varepsilon^{\mathrm{H}}=\varepsilon\left(\Sigma, P, \mathbb{C}^{*}\left(\varepsilon^{\mathrm{ef}}\right)\right)
$$

dont la résolution conduit à la valeur de $\varepsilon^{\text {er en fonction }}$ de $\Sigma$ et $\mathrm{P}: \varepsilon^{\mathrm{t}}=\mathrm{e}^{\mathrm{e}}(\Sigma, \mathrm{P}$ l. Introduisant cette détermination de er dans (65), on obtient la généralisation ron linéaire de (25)-(30):

$$
\begin{aligned}
& \Sigma=\mathbb{C}^{h}(\Sigma, P): \mathrm{E}-\mathrm{B}(\Sigma, P) P \\
& \phi-\phi_{0}=\mathrm{B}(\Sigma, P): \mathrm{E}+\frac{P}{N(\Sigma, P)}
\end{aligned}
$$

dans lesqueles l'expression de $\mathbf{B}(\Sigma, P)$ en fonction de $C^{\text {hom }}(\Sigma, P)$ alnsi que celle de $\Lambda(\Sigma$, P $)$ en fonction de $\mathrm{B}(\Sigma, \mathrm{P})$ sont formellement identiques à celles du cas lineaire:

$$
\begin{aligned}
& \mathrm{B}(\Sigma, P)=1:\left(\mathbb{I}-\mathbb{C}^{B}(\Sigma, P)^{-1}: \mathbb{C}^{\text {han }}(\Sigma, P)\right) \\
& \frac{1}{N(\Sigma, P)}=1: \mathbb{C}^{\sigma}(\Sigma, P)^{-1}:\left(-\varphi_{0} 1+\mathrm{B}(\Sigma, P)\right)
\end{aligned}
$$

\section{2}

\section{Application au cas isotrope}

Comme dans (60), on se place dans le cas isotrope, dans l"hypothèse où $\psi^{p}$ est une fonction de $\varepsilon_{x}$ et $\varepsilon_{\mathrm{f}}$. Dans l'esprit du concept de déformation effective, on adopte l'approximation $(64) \mathbb{C}^{s}(\varepsilon)=\mathbb{C}^{s}\left(\varepsilon^{e f}\right.$. On developpe ici un modèle isotrope oủ la déformation effective est caractérisée par deux invariants scalaires :

$$
\varepsilon^{e f}=\left(\varepsilon_{v}^{e f}, \varepsilon_{d}^{v f}\right) \quad ; \quad \varepsilon_{v}^{v f}=t r \bar{\varepsilon}^{s} ; \quad \varepsilon_{d}^{e f}=\sqrt{\bar{\varepsilon}_{d}^{s}}
$$

Lestimation approchée de l'élasticité du solide s'écrit donc:

$$
\mathbb{C}^{\delta}\left(\varepsilon^{e f}\right)=3 k^{s}\left(\varepsilon_{v}^{e f}, \varepsilon_{d}^{e f}\right) \mathbb{J}+2 \mu^{s}\left(\varepsilon_{v}^{e f}, \varepsilon_{d}^{e f}\right) \mathbb{K}
$$

Les quantités $\varepsilon_{i}^{\mathrm{ef}}$ et $\varepsilon_{\mathrm{r}}^{\mathrm{ef}}$ sont données en fonction de la contrainte macroscopique $\Sigma$ et de la pression de pore P par (45) et (55). Plus précisément, $E_{0}^{\text {tै }}$ dépend de $\Sigma+P$ $\phi_{0}$ l, tandis que $\varepsilon_{d}^{E f}$ est une fonction de $\Sigma+$ Pl. En vertu de (65). l'élasticité macroscopique $\mathbb{C}^{\text {hem }}$ de mène que
$\mathrm{B}$ et $\mathrm{N}$, wa dépendré á la fois de $\Sigma+\mathrm{P} \phi \mathbf{1}$ et de $\Sigma+$ P1. Ainsì, dans le cas général, la non-linéarté macroscopique ne saurait êtré contrôlée par un tenseur de contrainte effective (Domieux el al., 2002). Cependant, deปx exceptions mêritent d'être mentionnées :

- $k^{s}$ et $\mu^{5}$ ne dépendent pas de $\varepsilon_{\text {. }}$. Dans ce cas, (61) montre que $\mu^{3}$ est nécessairement constant. D'après (45), $\varepsilon^{\mathrm{e}}$ est déterminée a partir de la solution de

$$
\begin{array}{ll}
\left(1-\phi_{0}\right) \varepsilon_{v}^{-j}=\frac{1}{3 k^{j}} \operatorname{tr}\left(\Sigma+P \phi_{0} 1\right) & (u) \\
k^{-n}=k^{5}\left(\varepsilon_{v}^{-j}\right) & (\omega)
\end{array}
$$

$\varepsilon^{\mathrm{F}}$ apparat comme une fonction de $\Sigma_{m}+\mathrm{P}_{0}$ qui contröle donc la non-linéarité à l'échelle macroscopique (voir $(65))$ :

- $k^{5}$ et $\mu^{5}$ ne dépendent pas de $\mathrm{E}_{\mathrm{v}}$. Dans ce cas, la relation (61) montre que $k^{5}$ est nécessairement constant. En vertu de $(55), \varepsilon_{d}^{\text {ef }}$ est solutian de

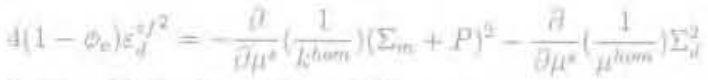

$$
\begin{aligned}
& k^{\text {ham }}=K\left(k^{*} \cdot \mu^{2}\right): \mu^{\text {hem }}=M\left(k^{*}, \mu^{2}\right) \\
& \mu^{\prime}=\mu^{*}\left(\varepsilon_{H}^{\prime}\right)
\end{aligned}
$$

Cette fois, la déformation $\mathrm{E}_{v}^{\text {ef }}$ est fonction de $\Sigma_{n}+\mathrm{P}$ et de $\Sigma_{a}$ et c'est la contrainte effective de Terzagh $\Sigma+$ P1 qui contrôle la non-linéarité du comportement à l'échelle macroscopique.

\section{Résistance d'un matériau poreux}

On suppose dans cette section que la résistance de la phase solide à l'óchelle microscopicue est caractérisëe par un critère de la forme $f^{3}(\sigma) \leq 0$. Le domaine $G^{5}$ des états de contraintes microscopiques compatibles avec les capacités de résistance du solide en question est défini par:

$$
\sigma \in G^{*} \Leftrightarrow I^{\prime}(\sigma) \leq 0
$$

\section{1}

\section{Rôle de la pression de pore sur la résistance macroscopique}

On s'intéresse pour commencer à l'influence de la pression de pore P sur la résistance macroscopique. Un état de contrainte et de pression $(\Sigma$, P) est dit admissible (du point de vue de la résistance) s'jl est possible de trouver un champ dé contrainte o[ $\mathrm{z}$ ) défini sur la phase solide du ver. et prolongé dans l'espace poreux qui soit compatible avec la résistance du solide au sens de (73), égal à $-\mathrm{P} 1$ dans $\Omega^{\mathrm{P}}$ et en équilibre avec $\Sigma$ au sens de la règle de moyenne sur les contraintes $\Sigma=\bar{\sigma}$. A la suite de de Buhan et Dormieux (1996), on introduit l'ensemble $\mathrm{G}^{\text {hom }}(\mathrm{P})$ des états de contrainte $\Sigma$ admissibles lorsque la pression de pore est fixée à la valeur $\mathrm{P}$

$$
\begin{aligned}
& \operatorname{civ} \sigma=0
\end{aligned}
$$

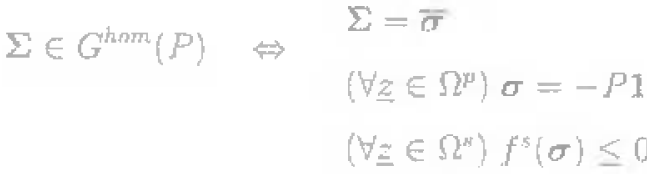


En particulier, $G^{\text {horn }}(0)$ est le domaine obtenu en l"absence de pression $(\mathrm{P}=0)$.

Considérons un état de contrairite macroscopique $\Sigma \in G^{\text {hom }}[\mathrm{P}]$ et un champ de contrainte microscopique $\sigma$ vérifiant (74). Introduisons $\bar{\sigma}=\sigma+$ Pl. On obtient :

$$
\Sigma \in G^{h a n}(P) \Leftrightarrow \exists \bar{\sigma}\left\{\begin{array}{l}
\operatorname{div} \tilde{\sigma}=0 \\
\Sigma+P 1=\overline{\bar{\sigma}} \\
\left(\forall \underline{\underline{z}} \in \Omega^{*}\right) \tilde{\sigma}=0 \\
\left(\forall \underline{z} \in \Omega^{*}\right) \bar{\sigma} \in G^{*}+P 1
\end{array}\right.
$$

où $\mathrm{G}^{5}+\mathrm{P} 1$ est l’image de $\mathrm{G}^{\mathrm{s}}$ dans la translation $\mathrm{a} \rightarrow \mathrm{a}+\mathrm{P} 1$

\section{2}

\section{Solide de von Mises ou de Tresca}

On fait d'abord l'hypothèse que la résistance de la phase solide n'est pas affectée par la contrainte moyenne. En d'autres temes. G est invariant dans les translations parallèlement à $1: \mathrm{G}^{\mathrm{h}}+\mathrm{P} 1=\mathrm{G}^{\mathrm{s}}$. Comme en (44), on scinde la contrainte microscopicue selon ses composantes sphérique et déviatorique :

$$
\sigma=\sigma_{d}+\sigma_{m} 1: \sigma_{m}=\frac{1}{3} \operatorname{tr} \sigma: \operatorname{tr} \sigma_{d}=0
$$

$\sigma_{m}$ est la contrainte moyenne et $\sigma_{\mathrm{d}}$ la partie déviatorique du tenseur de contrantinte. Dans la suite, on utilise également la contrainte déviatorique équivalente $\sigma_{\mathrm{f}}=$ $\sqrt{\sigma_{\mathrm{d}}: \sigma_{\mathrm{s}} / 2}$

Lorsque $\Sigma \in G^{\text {hom }(P) ~ l e s ~ p r o p r i e ́ t e ́ s ~ d u ~ c h a m p ~ o ̈ ~ d e ~}$ (75) assurent que $\Sigma+P 1 \in G^{\text {hom }}(0)$ :

$$
G^{\text {hut }}(0)=G^{\text {hem }}(P)+P 1
$$

Du encore :

$$
\Sigma \in G^{\text {ham }}(P) \Leftrightarrow \Sigma+P I \in G^{\text {Herr }}(0)
$$

En d"autres termes, le critère de résistance macros copique du milieı poreux saturé en présence de pression de pore peut être formulé en fonction de la contrainte effective de Terzaghi. Comme le montre [77). la détermination de $G^{\text {hom }}(P)$ pour une valeur quelconque de P revient à celle de $G^{\text {hom }}(0)$ : Gmom(P) est obtenu à partir de Gham(0) par une simple translation dans l'espace des contraintes (Fig. 3).

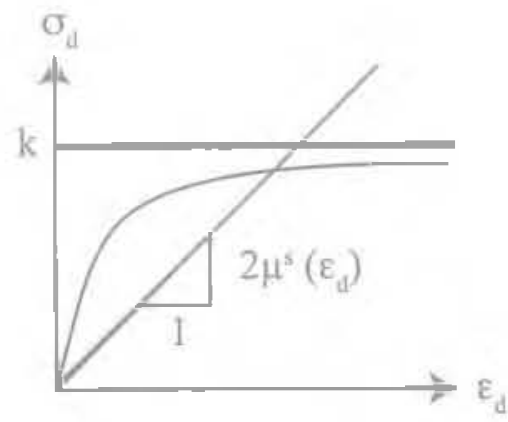

AG 3 Comportement non linéaire vérifiant la condition (80).

On expose à présent une méthode pour la détermination du critère de résistance macroscopique dans le cas ou le critèré de résistance de la phase solide est celui de von Mises et s'écrit donc:

$$
f^{n}\left(\sigma_{d}\right)=\frac{1}{2} \sigma_{d}: \sigma_{d}-k^{2}=\sigma_{d}^{2}-k^{2}
$$

Compte tenu du raisonnement précédent. il serait suffisant de se placer directement dans le cas d"un espace poreux non pressurisé $(\mathrm{P}=\mathrm{O})$. Cependant, on propose ci-après une approche directe de $G^{\text {hum }}(\mathrm{P})$ dont on vérifie qu'elle est bien en accord avec (77).

Le point de départ consiste à rechercher un matériau non linéaire, caractérisé par" un tenseur d'élasticité sécant $\mathbb{C}^{s}(\varepsilon)$, tel que la condition th $(\sigma)=0$ soit vérifiée asymptotiquement à l'échelle microscopique, pour une valeur suffisamment large de la déformation déviatorique locale $E_{d}$ :

$$
f^{3}\left(\lim _{x_{d} \rightarrow \infty} \sigma\right)=0
$$

La valeur de P étant fixée, on considère des trajets radiaux dans l'espace des déformations macroscopiques, de la forme : $\lambda \rightarrow \vec{\lambda} \overrightarrow{\mathrm{E}}$, la direction de $\overline{\mathrm{E}}$ étant arbitraire. On s'attenc à ce que de grandes déformations microscopiques soient induites localement pour des valeurs suffisamment élevées du paramètre $\lambda^{i \prime \prime}$. Dans ces conditions, l'état de contrainte macroscopique correspondant $\Sigma=\bar{\sigma}$ est situé sur la frontière de $\mathrm{G}^{\text {hom }}(\mathrm{P})$ :

$$
\lim _{\lambda \rightarrow \infty} \Sigma \in \partial G^{\text {hinmin }}(P)
$$

En d'autres termes, la frontiere $\partial G^{\text {hmm }}(P)$ est le lieu des extrémités des trajets de contraintes correspondant a des trajets de déformations radiaux. En revanche, pour les faibles valeurs de $\lambda$. l'état de contrainte macroscoplque reste à l'intérieur de Gum(P).

On cherche à défini le comportement non lineaire de la phase solide par uj potentiel $\Psi^{5}\left(E_{v}, E_{d}\right)$ de la forme.

$$
\psi^{*}\left(\varepsilon_{v}, \varepsilon_{d}\right)=\frac{1}{2} k^{*} E_{v}{ }^{2}+\mathcal{F}\left(\varepsilon_{d}\right)
$$

ou $k^{5}$ est une constante. $A$ partir de (82), la relation (59) conduit à :

$$
\sigma=k^{*} \varepsilon_{w} 1+2 \mu^{B}\left(\varepsilon_{d}\right) \varepsilon_{d} \text { avec } 2 \mu^{n}\left(\varepsilon_{d d}\right)=\frac{1}{2 \varepsilon_{d}} \mathcal{F}^{\prime}\left(\varepsilon_{d}\right)
$$

La condition [80) est satisfaite si $\sigma^{\prime}\left(E_{c}\right)=2 k$ pour des déformations déviatoriques suffisamment grandes. Cela conduit à choisir $\sim\left(\varepsilon_{\mathrm{d}}\right)=2 \mathrm{k} \varepsilon_{\mathrm{d}}$ dans cette gamme de déformation. En dautres tarmes, le module de cisaillement sécant $\mu^{5}\left(\varepsilon_{\mathrm{d}}\right)$ est une fonction décroissante de

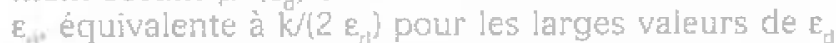
(Fig. 4). Asymptotiquement, on observe que $\mu^{5} \rightarrow 0$ tandis que $\mathrm{k}^{\mathrm{s}}$ reste constant. Cela implique que $\mu^{5} / \mathrm{k}^{5} \leq 1$, ce guł revient à dire que la phase solide se comporte formellement comme un matérau incompressible.

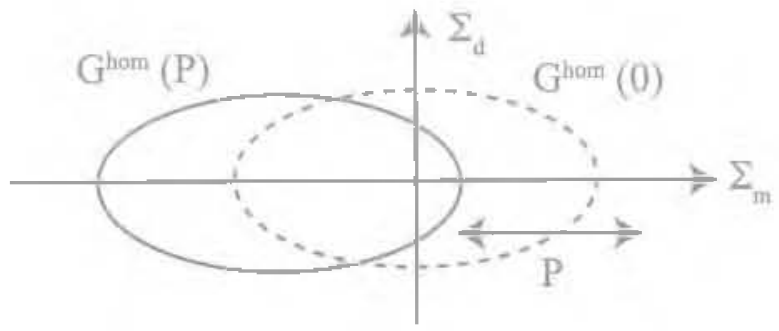

156. 4 Etats de contrainte macroscopiques admissibles pour $\mathrm{P}=0$ et $\mathrm{P} \neq \mathrm{0}$ (solide de von Mises).

On étudie maintenant la réponse du v.e.l: d'un milieu poreux dont la phase solide possede un tel com-

\footnotetext{
"11 Il est hon de noter qu'une déformation peut être grande par rapport a une déformation de réfërence, tout en demeurant dans le domaine des déformations infintésimales.
} 
portement. Il s'agit, comme on l'a vu à la section 3 , d'un problème d'homogénéisation norn linéaire, susceptible d'être appréhendé par une méthode sécante dans le cadre du concept de déformation effective. En vertu du fait que $k^{5}$ et $\mu^{5}$ ne dépendent pas de $\varepsilon_{\mathrm{v}}$, on est amené à résoudre l'équation (72). Sur le plan pratique, cela requiert de savoir decrire la dépendance de $k^{\text {hom }}$ et de $\mu^{\text {ham }}$ en fonction de $H^{5}$. On utilise à cet effet la borne supérieure de Hashin-Shtrikman comme estimation des modules macroscopiques. En vertu de la remarque sur l'incompressibilité de la plase solide, ces derniers s'écrivent (voir par exemple Dornieux 2005):

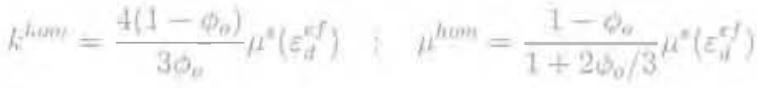
(72a) :

Ĺinvariant déviatorique effectif $\varepsilon_{u}^{e f}$ est déduit de $\left(2\left(1-\phi_{0}\right) \varepsilon_{d}^{4 /} \mu^{2}\left(\varepsilon_{N}^{2 /}\right)\right)^{2}=\frac{3 \phi_{u}}{4}\left(\Sigma_{n}+P\right)^{2}+\left(1+\frac{2}{3} \theta_{0}\right) \Sigma_{d}^{2}$

En utilisant le fait que $\mu^{\mathrm{w}}\left(\mathrm{E}_{\mathrm{g}}\right) \approx \mathrm{k} /\left(2 \varepsilon_{\mathrm{d}}\right)$ dans le domaire des grandes déformations, on obtient que l'état de contrainte macroscopique asymptotique est situé sur une ellipse du plan $\left(\Sigma_{m,} \Sigma_{d}\right)$ :

$$
\frac{3 \phi_{o}}{4\left(1-\phi_{0}\right)^{2}}\left(\Sigma_{m}+P\right)^{2}+\frac{1+2 \phi_{o} / 3}{\left(1-\phi_{o}\right)^{2}} \Sigma_{d}^{2}=k^{2}
$$

On retrouve au passage le fait que la résistance macroscopique est contrôlée par la contrainte effective de Terzaghi $\mathrm{L}+\mathrm{P} 1$

\section{3}

\section{Solide de Drucker-Prager}

La conclusion qui vient d"ètre tírée dans le contexte des matériaux de von Mises n'est plus valable lorsque le criterre de la phase solide est sensible à la contrainte moyenne. A titre d'illustration, on fait l'hypothèse que le domaine $\mathrm{G}^{5}$ des contraintes admissibles pour la phase solide est un cône. Son sommet est situé sur la droite $\sigma_{1}=\sigma_{2}=\sigma_{3}$ de l'espace des contraintes principales et represente un état de traction isotrope $\mathrm{h} 1$. Par référence à la valeur de la résistance en traction Isotrope $\mathrm{b}, \mathrm{G}^{5}$ sera noté $\mathrm{G}_{\mathrm{n}}^{\mathrm{s}}$. L'ensemble des états de contrainte macroscopiques admissibles en l'absence de pression est note $G_{h}^{\text {hwm }}(0)$.

Par exemple, le matériau de Drucker-Prager (Fig. 5) dont le critère s'écrit:

$$
\begin{gathered}
f^{\prime \prime}(\sigma)=a\left(\frac{1}{3} \operatorname{tr} \sigma-h\right)+ \\
\sqrt{\frac{1}{2} \sigma_{d}: \sigma_{d}}=a\left(\sigma_{m}-h\right)+\sigma_{d} \leq 0
\end{gathered}
$$

appartient manifestement à cette classe de matériaux.

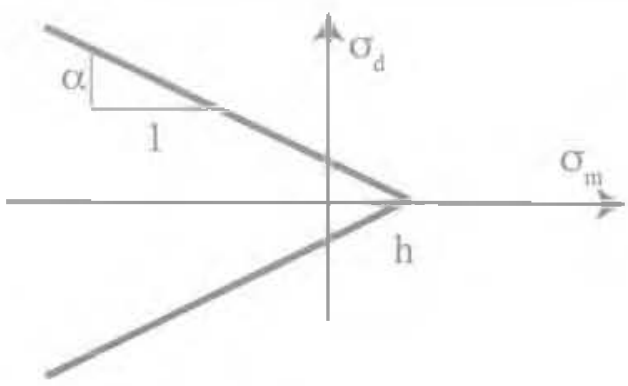

FG.s Critère de Drucker-Prager: états de contrainte microscopiques admissibles.
Il est utile d'observer que les ensembles $G^{5}$ et $G^{\mathrm{s}}$. associés à deux valeurs différentes het h' de la résistance en traction isotrope peuvent être déduits l'un de l'autre par une translation, ou encore par une homow thétie ${ }^{\lfloor 2\rfloor}$ :

$$
\text { (a) } G_{h^{\prime}}^{*}=\frac{h^{\prime}}{h} G_{h}^{*} \quad \text { (b) } \quad G_{h^{\prime}}^{*}=G_{h}^{\prime}+\left(h^{\prime}-h\right) 1
$$

En vertu de (88a) et de la définition de G Gom donnée en ( 74 ) (avec $P=0$ ), on en déduit que $G$ hom (0) dépend linéadrement de h:

$$
G_{h^{\prime}}^{\text {hem }}(0)=\frac{h^{\prime}}{h_{h}} G_{h}^{\text {hiam }}(0)
$$

Par ailleurs, il est facile de voir à partir de [88b) que (Fig. 6)

$$
G_{h}^{s}+P 1=G_{h+P}^{*}
$$

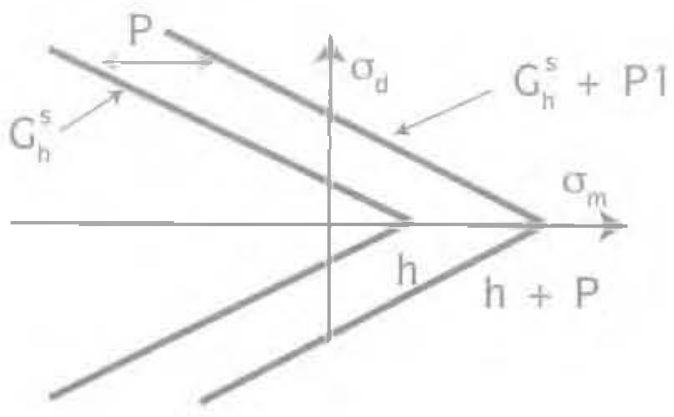

मG. $6 \mathrm{G}_{\mathrm{h}}^{s}$ et $\mathrm{G}_{\mathrm{h}}^{s}+\mathrm{P} 1=\mathrm{G}_{h+\mathrm{p}}^{s}$ dans le cas du critère de Drucker-Prager.

L'utilisation de ce résultat dans (75) montre que:

$$
\Sigma \in G_{h}^{\text {horn }}(P) \Leftrightarrow \Sigma+P 1 \in G_{h+P}^{h a m}(0)
$$

La combinaison de (89) et de (91) permet finalement d'affirmer" que :

$$
G_{h}^{\text {han }}(P)+P 1=\left(1+\frac{P}{T_{l}}\right) G_{h}^{\text {hom }}(0)
$$

oL eñcore:

$$
\Sigma \in G_{h}^{h o m}(P) \Leftrightarrow \frac{\Sigma+P 1}{1+P / h} \in G_{h}^{h o m}(\theta)
$$

Cela signifie que la rêsistance macroscopicue est contrôlée par la contrainte effective suivante:

$$
\Sigma^{\prime \prime}=\frac{\Sigma+P 1}{I+P / h}
$$

A la différence du concept traditionnel de contrainte effective, on rote que $\Sigma$ " ne dépend pas linéairement de la pression de pore P. D'un point de vue géométrique, la relation (92) exprime que Ghom (P) peut être céteminé ả partir de $\mathrm{G}_{\mathrm{h}}^{\mathrm{kn}}(0)$ par l'application de deux transformations consëcutives. En premier lieu, l'homothétie de rapport $1+\mathrm{P} / \mathrm{h}$ est appliquée à $\mathrm{G}_{\mathrm{h}}^{\text {hom }}(0)$ suivie de la translation $\Sigma \rightarrow \Sigma-$ P1 (Fig. 7 ).

2. h. $G^{\mathrm{s}}$ désigne l'jmage de Gs par l'homothétie dont le centre est sítué à l'origine, et dont le rapport est égal à $\lambda_{:}: z \rightarrow \lambda_{2}$. 


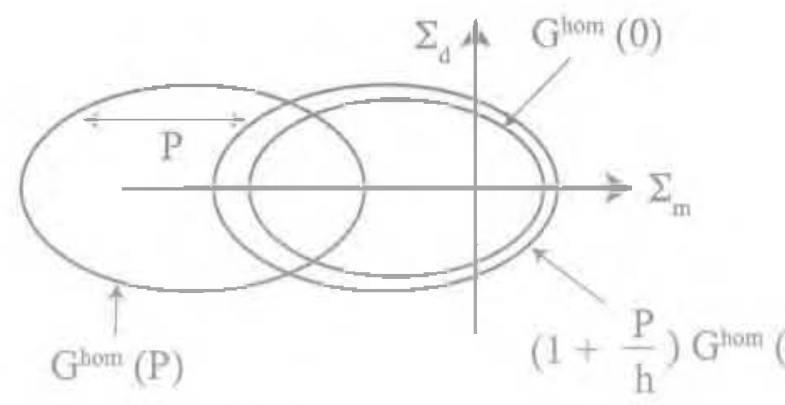

FIG. 7 Soliqe de Drucker-Prager: étals de contratinte macroscopiques admissibles en présence et en l'absernce de pression de pore.

On considère, à titre d'exemple, le cas particulier où la phase solide est un matériat de Drucker-Prager (87). Puisque l'existence d'une contrainte effective macroscopique a pu être établie, il est suffisant de dëterminer la résistance macroscopique en l'absence de pression de pore $(P=0)$, ce quil revient à identifier le domaine $G_{h}^{\text {hnm }}(0)$. G $G_{h}^{\text {hom }}(P)$ sera alors déduit de (93). On utilise cet effet la technique mise en ouvre précédemment dans le cas du matériau de von Mises. On recherche donc un matériau isotrope non linéaje cont l'équation d'étatest de la forme (58) et vérifiant la condition (80):

$$
\mu^{\sigma}\left(\varepsilon_{v}, \varepsilon_{d}\right) \approx \frac{\alpha}{2 \varepsilon_{d}}\left(h-k^{*} \varepsilon_{y}\right)
$$

dans te domaine des grandes déformations déviatoriques. A la différence du module de cisaillement identifié dans le cas du matéríau de von Mises $\left(\mu^{5}\left\langle\varepsilon_{d}\right)=k /\right.$ $\left(2 \varepsilon_{t}\right)$. le module de cisaillement secant $\mu^{\text {s }}$ daris 195 ? dépend à la fois de $\mathrm{e}_{\mathrm{y}}$ et de $\varepsilon_{\mathrm{d}}$. Cette propriété est destinée à traduire le fait que la résistance au cisaillement. représentée par $2 \jmath^{5} \varepsilon_{d}$ augmente avec la pression de confinement locale qui n'est autre que - $k^{s} \varepsilon_{\mathrm{y}^{\prime}}$ En ce qui concerne le module de compression $k^{s}$, le choix le plus simple consiste à le considérer comme une constante. On remarque qu'une telle définition ne permet pas de satisfaire la condition (61) assurant l'existence d'ur potentiel. Notons que ce dernier n'est cependant pas nécessaire pour mettre en ceuvre le raisonnement. Le miveau de déformation effectif est ici caractérisé par les deux scalaires $\mathrm{E}_{u}^{\mathrm{l}}$ et $\boldsymbol{\varepsilon}_{\text {el. }}$. Avec les relations (45) et (55), compte tenu de la condition $\mathrm{P}=0$, le systême qui per" nuet de déterminer ces quantités en fonction de l'étal de contrainte macroscopique est le suivant

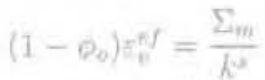

$$
\begin{aligned}
& 4\left(1-\theta_{o}\right) \Sigma_{d}^{2} \gamma^{2}=-\frac{\partial}{\partial \mu^{n}}\left(\frac{1}{\mu^{h i n m}}\right) \Sigma_{m}^{2}-\frac{\partial}{\partial \mu^{2}}\left(\frac{1}{\mu^{h+m}}\right) \Sigma_{d}^{2} \\
& k^{\text {hem }}=\frac{4\left(1-\phi_{0}\right)}{3 \phi_{i}} \mu^{*} ; \quad \mu^{\text {himm }}=\frac{1-\phi_{o}}{1+2 \phi_{0} / 3} \mu^{*}
\end{aligned}
$$

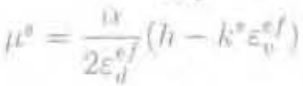

Comme dans le cas du solide de von Mises, on a utilisé dans (96c) le fait que $\mu^{\mathrm{s} /} / \mathrm{k}^{\mathrm{s}}$ tend vers 0 pour les grandes déformations déviatoriçues. En combinant (96b) et (96c), il vient tout d'abord :

$$
\left(2\left(1-\phi_{o}\right) \varepsilon_{d}^{e f} \mu^{\mu}\left(\varepsilon_{d}^{e f}\right)\right)^{2}=\frac{3 \phi_{o}}{4} \Sigma_{m}^{2}+\left(1+\frac{2}{3} \phi_{o}\right) \Sigma_{d}^{2}
$$

II reste à introduire (96a) et (96d) dans le membre de gauche de (97) :

$$
\begin{gathered}
\alpha^{2} h^{2}\left(1-\phi_{0}\right)^{2}=\left(\frac{3 \phi_{o}}{4}-\alpha^{2}\right) \Sigma_{m}^{2}+ \\
\left(1+\frac{2}{3} \phi_{o}\right) \Sigma_{d}^{2}+2 \alpha^{2} h\left(1-\phi_{0}\right) \Sigma_{m}
\end{gathered}
$$

La relation (98) caractérise le domaine $G_{1}^{\text {hom }}(0)$, Ensuite, Gur (P) est obtenu en remplaçant l'état de contrainte macroscopique $\Sigma$ par la contrainte effective (94). Avec les motations

$$
\Sigma_{m}^{\prime \prime}=\frac{\Sigma_{m}+P}{1+P / h} \quad ; \quad \Sigma_{d}^{\prime \prime}=\frac{\Sigma_{d}}{1+P / h}
$$

I'équation de la frontière elliptique de Ghom (P) dans l'espace des contraintes $\left(\Sigma_{m}^{\prime \prime}, \Sigma_{m}^{\prime \prime}\right)$ s'écrit:

$$
\begin{aligned}
& a^{2} h^{2}\left(1-\phi_{o}\right)^{2}=\left(\frac{3 \phi_{e}}{4}-a^{2}\right) \Sigma_{m}^{\prime \prime 2}+ \\
& \left(1+\frac{2}{3} \phi_{o}\right) \Sigma_{d}^{\prime \prime 2}+2 a^{2} h\left(1-\phi_{e}\right) \Sigma_{m}^{\prime \prime}
\end{aligned}
$$

Notons que l'on retrouve [86] comme la limite de (100) quand $\mathrm{h} \rightarrow$ - et $\alpha \rightarrow 0$ avec $\alpha h=\mathrm{k}$.

Supposons à nouveau que $\mathrm{P}=0$. D'après l'équation (98), la forme de $G_{n}$ (0) est effectivement elliptique et la résistance en compression isotrope est bonée à la condition que $3 \phi / 4>\alpha^{2}$. En revanche, pour de plus fajbles valeurs de la porosité, en l'occurrence pour $3 \phi_{c} / 4$ $<\alpha^{2}$, il convient d'observer que la relation (98) prédit gue la résistance en compression isotrope est infinie. En d'autres termes, pour de telles porosités, tout état de contrainte de la forme $\Sigma 1$ avec $\Sigma<0$ est admissible, de sorte que $\mathrm{G}_{\mathrm{h}}^{\text {har }}$ (0) n est pas un domaine fermé. Cette conclusion est en contradiction avec le lésultat du modèle unidimensionnel de la sphère creuse sous pression (Dormieux et al., 2006). On montre en effet que la contrainte de confinement qui peut être appliquée à la sphère creuse est bornée, quelle que soit sa porosité (pourvu que $\alpha<\sqrt{3} / 2$ ). Plus précisément, la valeur exacte $\Sigma^{+}$de la contrainte de confinement maximale peut être obtenue par les techniques classiques du calcul a la rupture et vaut (Barthélémy et Dornieux, 2003):

$$
\Sigma^{+}=h\left(\phi_{0}^{\frac{2 n / \sqrt{5}}{1-2 a / \sqrt{3}}}-1\right)
$$

Cette limitation du résultat du processus d'homo gênéisation nom liméaire est imputable au manque de précision dans la mise en oeuvre du concept de déformation effective. Le recours à une estimation uniforme du riveau de déformation sur l'intégralité de la phase solide devient trop simpliste pour les basses valeurs de la porosité. Cette difficulté peut cependant être sumontéc en subdivisant le domaine solide en sousdomaines, en introduisant autant de deformations effectives que de sous-domaines.

Une alternative à la méthode d’homogénéisation non linéaire conduisant aux critères de rupture (98) et (100) consiste à mettre en cuve une technicule d"homogénéisation périodique. La confrontation des deux méthodes permet égajement d'apprécier les performances quantitatves des expressions analytiques très simples fournies par la méthode sécante dans le cadre du concept de déformation effective.

On considère ici une cellule de base cubique, comportan! une sphere creuse au centre, entourée de la phase solide. On a vu qu'il n'est pas restrictif de supposer la pression de fluide nulle. Le milieu périodique est obtenu par répétition dans l'espace de ce motif 
de base. On détermine la charge limite de ce dernier lorsqu'ih est soumis à trajet radial dans l'espace dos contraintes. Dans la pratique, on se limite ici à des états triaxiaux de révolution dars les axes de la celluje et l'on fait varier le rapport $\Sigma_{\mathrm{d}} / \Sigma_{\mathrm{m}}$,

La figure 8 présente une comparaison entre le critere de rupture (98) (courbe en trait continu) et les résultats numériques obtenus par un calcul en éléments finis (cercies), pour les valeurs $\alpha=0,21$ et $\phi_{4}=$ 0,15. Les segments inclinés de part et d'autre de l'axe vertical correspondent aux trajets de compressjon et de traction untaxiales. Sur ce diagramme, on note les deux trajets rectilignes radiaux qui correspondent à des états de compression et de traction simples. On observe un excellent accord pour des niveaux de contratnte moyenne égaux ou inférieurs à celui de l'état de compression simple. En revanche, le résultat du schéma d'homogénélsation non lineaire surestime significativement la résistance pour les états de contrainte en compression faiblement déviatoriques. On retrouve les limites de cette démarche qui viennent d'étre commentées à propos de la résistance en compression isotrope.

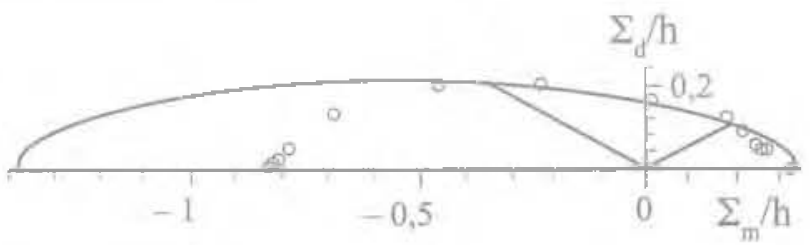

flG.7 Critêre de rupture macroscopique pour une phase solide de type Drücker-Prager: approche périodique el estimation par homogénéisation non linéaire.

\section{Conclusion}

Parallèlement ì l'appoche expérimentale, la démaŕche micromécanigue constitue une stratégie efficace pour tester l'existence d'une contrainte effective dans un cadre rhéologique donné. Bien qu'elie soit couramment admise pour les sols et largement validée par de nombrewx essais de laboratoire, la contrainte effective de Terzaglii ría pas de portée qérérale. D'une façn bien connue en Mécanique des Roches, la contrainte effective proposée par Biot dans le cadre de la poroêlasticité linéaire doit lui être préférée. La question ce l'existence d'une contrinte effective dans les situations de comportement non linéaire, élastique ou non. est plus ouverte. S'agissant du critère de rupture, la contrainte de Terzaghi est attachée à l'absence d'effet du confinement sur la résistance de la phase solide du milieu poreux. Le recours à une contrainte effective plus complexe, variant de façon non lineaire en fonction de la pression, s'impose en revanche lorsque la phase solide du milieu poreux posséde une sensibilité au confinement de type Drüker-Prager.

Il est à noter qu'un êcart vis-à-vis de la contralnte de Terzaghi peut également résulter d’interactions non purement mécaniques entre les constituants de

la phase solide à l'échelle microscopique. C'est le cas notamment dans les argiles de la classe des smectites (Dormieux et al., 2005).

Signalons pour finir quelques résultats relatifs aux matériaux élastiques linéaires endommagés par microfissuration. On suppose que le réseau de fissures est saturé et connecté, de sorte qu'il y règne une pression uniforme. Dans le domaine des évolutions réversibles, on montre que la refermeture progressive des fissures se traduit par un comportement poroélastique non linéaire. Les équations d'état s'êcrivent de façon commode en vitesse, en faisant appel à des coefficients de poroélisticité tangents :

$$
\begin{aligned}
& \Sigma=\mathbb{C}_{\text {l.sin }}^{\text {lom }}(\Sigma+P 1): \dot{\mathbf{E}}-\mathbf{B}_{\text {ray }}(\Sigma+P 1) P \\
& \dot{\phi}=\mathrm{B}_{\tan }(\Sigma+P 1)=\dot{\mathrm{E}}+\frac{\hat{P}}{\hat{N}_{\operatorname{lan}}(\Sigma+P 1)}
\end{aligned}
$$

De surcroit, ces derniers dépendent de la contrainte effective de Terzaghi (Dormieux et Kondo, 2005). Sous réserve de l'homogénétté de la phase solice, ils sont reliés par les relations homologues de (26) et (31).

Enfin, signalons que le modèle micromécanique de l'évolution de l"endommagement par microfissuration proposé récemment (Dormieux et al., 2006a) conduit à un critere de propagation également contrôlé par la contrainte effective de Terzaghi.

\section{1}

\section{Annexe : le lemme de Hill}

On considère un champ de contrainte $\sigma$ à divergence nulle et un champ de déformation géonétriquement compatible $e^{*}$ définis sur le v.e.r. $\Omega$. On envisage deux situations distinctes

- le champ de contrainte $\sigma^{\prime}$, de moyenne $\Sigma^{\prime}=\overrightarrow{\sigma^{\prime}}$, vérifie des conditions aux limites uniformes en contrainte

$$
(\forall z \in \partial \Omega) \sigma^{\prime}(z) \cdot \underline{n}(z)=\Sigma \cdot \underline{n}(\underline{z})
$$

- le champ de déformation $\varepsilon^{*}$, de moyenne $\mathrm{E}=\overline{\mathrm{E}^{*}}$, est cinématiquement admissible avec un champ de déplacement $\xi^{*}$ verifiant des conditions alux limites uniformes en déformations

$$
(\nabla \underline{z} \in \partial \Omega) \xi(z)=\mathbb{E}^{*} \cdot \underline{z}
$$

Dans les deux cas, on démontre la régle de moyenne suivante:

$$
\overline{\sigma^{\prime \prime}: \varepsilon^{*}}=\overline{\sigma^{*}}: \overline{\varepsilon^{*}}
$$

Ge résultat constitue le lemme de Hill. Il garantit lé cohérence du changement d'échelle sur le plan énergétique.

\section{AEMIIRCIEMINTS}

Lauteur thent à cemencier Samir Maghum pour de fructueuses discus-

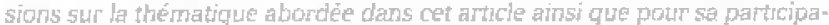

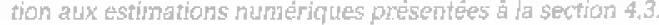




\section{$\overline{\text { Bibliographie }}$}

Auriault J.L., Sanchez-Palencia E. - Étude du comportement macroscopique d'un milieu poreux saturé déformable. Journal de Mécanique, "16, 1977, p. 575-603.

Barthélémy J.F., Dornieux L. - Détermi. nation du critềre de moture macroscopique d"un milíe poreux par homogé. néisation non linéaire. C.P. Mécanique, 331, 2003, p. 77-84

Bishop A.W. - The principle of effective stress. Norwegian Geotechnical institute Publication. 32. 1960, p. 1-5.

Buhan (de) P. Dormievx L. - Cn the validity of the effective stress concept for assessing the strength of saturated porous materials : a homogenization approach. J. Mech. Phys, Solids. 44 . 1996, p. $1649-1667$

Chateau X., Dornieux L - Micromechanics of saturated and unsaturated porous medie. Int. I. Numer. Anes. Methods Geonech. 26, 2002, p. 831-844.

Coussy 0. - Mechanics of porous conthua. Wiley, 1955.
Domieux L. - Poroelasticity and strength of fully or partially saturated porous materials. Applied micromechanics of porous materials l"L. Dormieux and F.-J. Ulm, ed.). CHSM Courses and Lectures $\pi^{\circ} 480$, Springer, 2005

Dormieus L, Kondo D. - Poroelasticity and damage theory for saturated cracked media. Applied micromechanics of porous materials (L. Dormieuse and EJ) Lim, ed.. CISM Courses and Lectures $\pi^{\circ} 480$. Springer, 2005.

Domienx R., Kondo D., UIm F.J. - A micromechanical analysis of damage propagation in fluid-saturated cracked media. C.R. Mecanigue 334, 2006, p. 440 446

Dormieux Lu, Kondo D. F.J. Uim. Microporomechanics. Wiley, 2006

Dormieux L., Lemarchand E., Sanahuja J. - Comportement macroscopique de materieux à microstructure en feuillets. Microstructure et Propriétés des MaléTiaux. Presses de l'ENPC. 2005

Dormieux I.. Molinari A.. Kondo D. Micromechanical approach po the behaw- ior of poroelastic materials I Mech. Phys. Solkds, 50, 2002, p.2203-2231.

Fredlund D.G. - Soil mechanics principles that embrace unsaturated solls. Proc of the 11th International Conference an Soil Mechanics and Foundation Enginemering. vol. 2. 1985, p. 465-472.

Kreher W. - Residual stresses and stored elastic enercy of composites and polycristals. J. Mech. Fhys Solids, 38, 1990 p. $115-128$.

Schrefter B., Simoni L. Li X., Zjenklewicz O.C. - Mechanics of partially saturated porous nodia. Mumerica methods and constitutiwe modelling in geomechanics (C.S. Desai and Gioda, ed.j. CISM Courses and Lectures no 311 . Springer, 1997

Suguet P. - Effective hehavior of non linear composites. Continum mitromechanics (P. Suquet, ed I. CISM Courses and Lecures $\Pi^{\circ} 377$. Springer. 7997

Zaoui A. - Continum mímromechanics: survey. It. Eng. Mech., 128 [8], 2002. p. 808 -816. 